\title{
Implantation Studies on Silicon-Doped GaN
}

\author{
RONNIE SIMON, ${ }^{1,3}$ REINER VIANDEN, ${ }^{1,4}$ and KLAUS KÖHLER ${ }^{2}$ \\ 1.-Helmholtz-Institut für Strahlen- und Kernphysik, 53115 Bonn, Germany. 2.-Fraunhofer \\ Institut für angewandte Festkörperphysik (IAF), 79108 Freiburg, Germany. 3.-JCNS-2, \\ PGI-4, Forschungszentrum Jülich GmbH, 52425 Jülich, Germany. 4.—e-mail: vianden@hiskp. \\ uni-bonn.de
}

\begin{abstract}
Silicon-doped GaN layers grown by low-pressure metalorganic vapor-phase epitaxy with $\mathrm{Si}$ concentrations ranging from $2 \times 10^{17} \mathrm{Si} / \mathrm{cm}^{3}$ to $9.2 \times 10^{18}$ $\mathrm{Si} / \mathrm{cm}^{3}$ were investigated by means of the perturbed angular correlation (PAC) technique applied to implanted ${ }^{111} \mathrm{In}(\mathrm{Cd})$. An undoped $\mathrm{GaN}$ film is used as a reference. The $\mathrm{Si}$ atoms replace $\mathrm{Ga}$ atoms in the lattice, and silicon, being a group IV element, acts as a donor on the Ga site and contributes one extra electron to the conduction band. Hall-effect measurements confirmed that the free charge carrier density is essentially increased and of the order of the silicon concentration. PAC investigations of the annealing behavior after implantation of the ${ }^{111}$ In probes show that best recovery is achieved after annealing at $1200 \mathrm{~K}$ and that high silicon concentrations make GaN films more stable at high temperatures. Further, it was found that the temperature dependence of the electric field gradient is reduced by increasing Si concentrations.
\end{abstract}

Key words: Nitride semiconductor, implantation, annealing, perturbed angular correlation

\section{INTRODUCTION}

Group III nitride semiconductors, especially gallium nitride $(\mathrm{GaN})$, have drawn much attention in recent years. One remarkable feature of these materials is the formation of continuous alloy systems, e.g., $\operatorname{In}_{x} \mathrm{Ga}_{1-x} \mathrm{~N}$, in which the optical bandgap can be tailored by varying the concentrations of the constituents. This makes group III nitrides ideal for optoelectronic devices. In order to improve the possibilities of structuring $\mathrm{GaN}$ devices it is desirable to dope the material by ion implantation. Then, however, lattice damage is created and has to be annealed by an appropriate temperature program. Thus, in this work the effect of doping on the annealing behavior after ion implantation and stability of GaN films at high temperatures is investigated. Further, the influence of changes in the charge carrier density on the hyperfine fields in $\mathrm{GaN}$ and their temperature dependence is studied. A nuclear technique, namely the perturbed angular correlation technique, is employed in this work.

(Received June 26, 2012; accepted September 18, 2012; published online October 20, 2012)

\section{EXPERIMENTAL PROCEDURES}

The perturbed angular correlation (PAC) method is an experimental technique of nuclear solid-state physics. It is based on the hyperfine interaction between a radioactive probe atom and fields present at the position of the probe atom. In more detail, the nuclear electric quadrupole and magnetic dipole moment interact with electric field gradients (EFG) and magnetic fields, respectively. In solids, an EFG can originate from the charge distribution in a noncubic crystal lattice such as the wurtzite structure found in many group III nitride semiconductors. Crystal defects such as substitutional and interstitial impurities or vacancies also lead to an EFG. Thus, lattice damage, which unavoidably occurs during the doping of semiconductors by ion implantation, can be investigated in detail by PAC.

Experimentally, the hyperfine interaction is observed in the time-dependent change of the angular correlation between two successive $\gamma$-rays in a $\gamma-\gamma$ cascade due to an external perturbation by an EFG. A comprehensive description of the theory of perturbed angular correlation can be found in the literature. $^{1}$ 
Table I. Silicon concentration and thickness of the doped layer measured with SIMS

\begin{tabular}{lcc}
\hline $\begin{array}{c}\text { Sample } \\
\text { Number }\end{array}$ & $\begin{array}{c}\text { Si Concentration } \\
\left(\mathbf{a t o m s} / \mathbf{c m}^{\mathbf{3}}\right)\end{array}$ & $\begin{array}{c}\text { Layer Thickness } \\
(\mathbf{n m})\end{array}$ \\
\cline { 2 - 2 } GN 5465 & Undoped & 2,400 \\
GN 5499 & $2.0 \times 10^{17}$ & 890 \\
GN 5895 & $6.0 \times 10^{17}$ & 980 \\
GN 4877 & $1.1 \times 10^{18}$ & 900 \\
GN 4876 & $3.7 \times 10^{18}$ & 900 \\
GN 4885 & $9.2 \times 10^{18}$ & 970 \\
\hline
\end{tabular}

In the present experiment, the angular correlation of the $172 \mathrm{keV}-245 \mathrm{keV} \gamma-\gamma$ cascade emitted in the decay of the PAC probe ${ }^{111} \mathrm{In}$ to ${ }^{111} \mathrm{Cd}$ was measured in conventional fast-slow PAC setups equipped with $\mathrm{BaF}_{2}$ or lutetium oxyorthosilicate (LSO) scintillators coupled to fast photomultipliers. Further details on these two systems can be found in the PhD theses of $\mathrm{Koch}^{2}$ and Valentini. ${ }^{3}$ From the data, the quadrupole interaction (QI) frequency $v=e Q V_{z z} / h$ as well as the symmetry of the EFG $(\eta)$ and its orientation can be derived. Any damping of the QI frequency gives information on remaining defects near the probe atoms. The fraction of

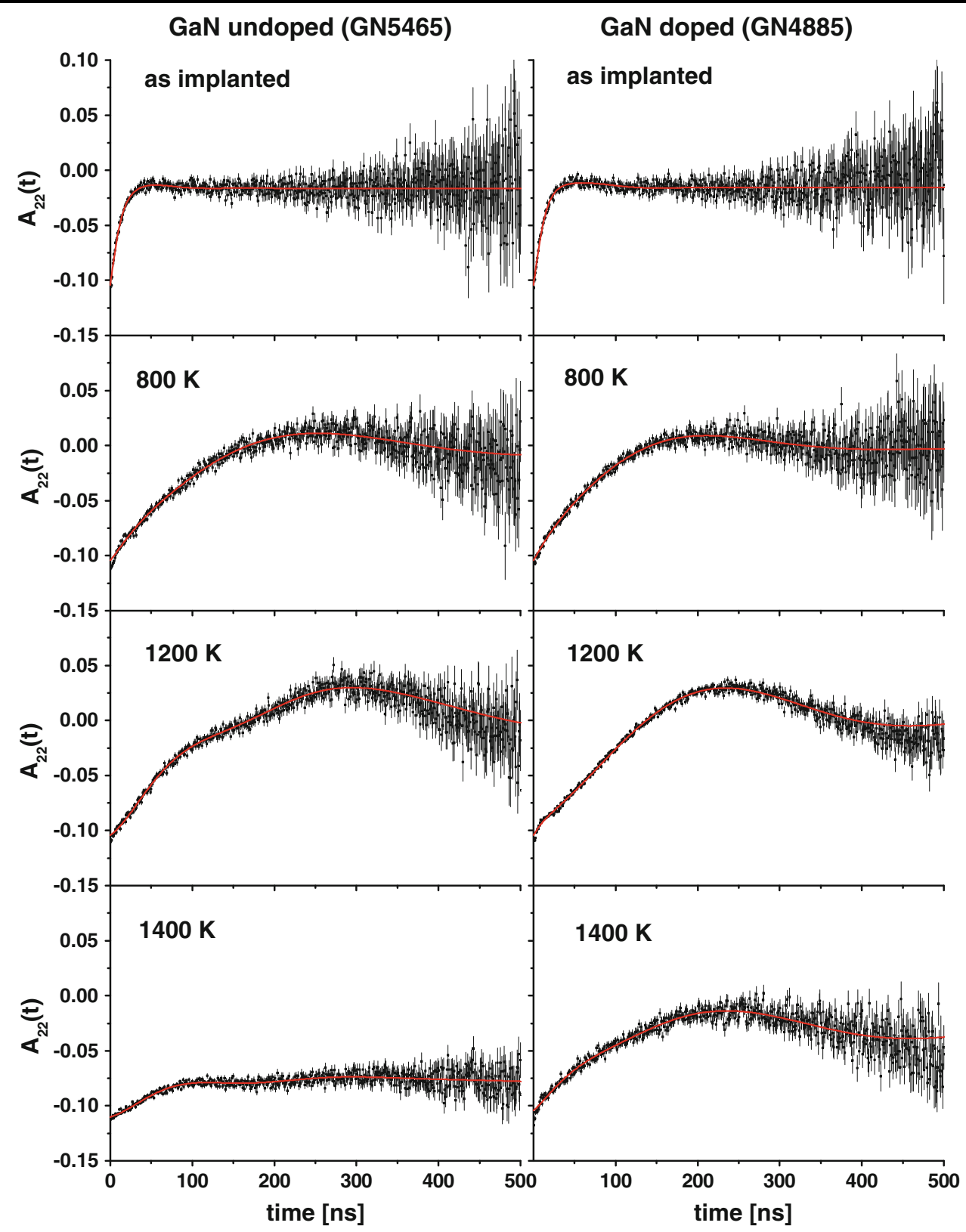

Fig. 1. Time-dependent anisotropy $A_{22}(t)$ for ${ }^{111}$ In after implantation into GaN samples with different Si doping levels (undoped: left side; $9.2 \times 10^{18} \mathrm{Si} / \mathrm{cm}^{3}$ doped: right side). The $c$-axis of the crystal is aligned at an angle of $45^{\circ}$ relative to the start detector in the detector plane. Top to bottom: spectra obtained after annealing for $120 \mathrm{~s}$ under nitrogen at the temperature indicated in the frames. 


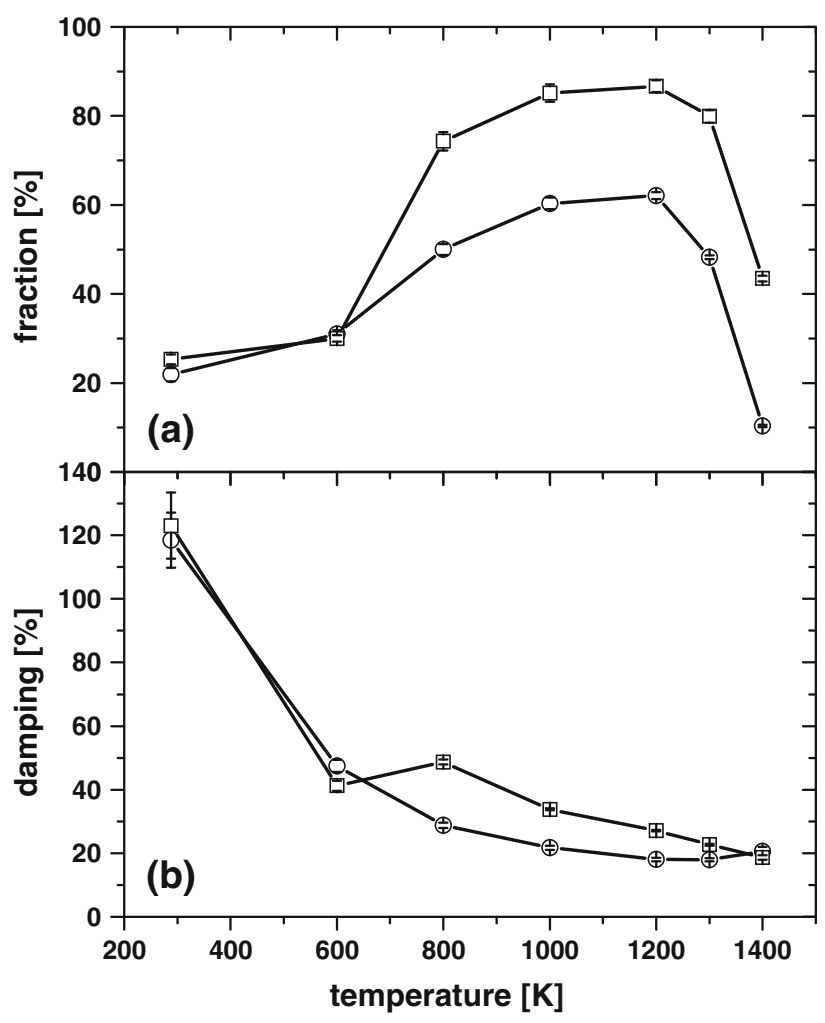

Fig. 2. (a) Fractions of In probe atoms in GaN on regular lattice sites after annealing at different temperatures and (b) the width of the frequency distribution around the lattice QI frequency (open squares = highest $\mathrm{Si}$ doping of $9.2 \times 10^{18} / \mathrm{cm}^{3}$; open circles = undoped).

implanted atoms in the same environment is directly proportional to the amplitude of the respective QI pattern in the $A_{22}(t)$ spectra.

\section{SAMPLE PREPARATION}

All samples investigated in this study were grown by metalorganic vapor-phase epitaxy (MOVPE) on 2-inch sapphire substrates in $c$-direction, $\mathrm{Ga}$ face. The layer sequence consists of a $25-\mathrm{nm}$ GaN lowtemperature nucleation layer followed by an undoped $2-\mu \mathrm{m} \mathrm{GaN}$ buffer layer, in order to improve the crystal quality. On top of that, silicon-doped GaN layers with different thicknesses ranging from $890 \mathrm{~nm}$ to $970 \mathrm{~nm}$ were grown. For this study, silicon-doped GaN films with five different concentrations ranging from $2 \times 10^{17} \mathrm{Si} / \mathrm{cm}^{3}$ to $9.2 \times 10^{18} \mathrm{Si} / \mathrm{cm}^{3}$ were available. Additionally, an undoped GaN film (GN 5465) of $2.4 \mu \mathrm{m}$ thickness is used as a reference. The silicon concentration and thickness of the doped layer were measured using secondary-ion mass spectrometry (SIMS). Further details about the sample properties can be found in Table I and in an article of Köhler et al. ${ }^{4}$

Implantation of ${ }^{111}$ In PAC probe atoms was carried out at the Bonn Isotope Separator. To prevent channeling of the implanted ions, the samples were tilted by an angle of $7^{\circ}$ with respect to the ion beam direction. According to previous studies in our group, no channeling effects are to be expected in this configuration. The implantation energy was set to $160 \mathrm{keV}$, and a typical dose of $1 \times 10^{12} \mathrm{In} / \mathrm{cm}^{2}$ was reached. The mean implantation depth is simulated to be $399 \AA$, the full-width at half-maximum (FWHM) of the implantation profile to be $158 \AA$, and the maximum implantation depth to be $1163 \AA^{5}{ }^{5}$ Comparing the maximum implantation depth with the thickness of the doped layers (Table I), it is obvious that the indium probes do not reach the GaN buffer layer beneath the silicon-doped layer.

Annealing steps were carried out under nitrogen atmosphere between carbon strips in a rapid thermal annealing setup. To exclude variations in the annealing conditions, normally two samples were annealed together face to face, thus serving as a proximity cap to each other. For measurements at elevated sample temperatures a PAC furnace was used. ${ }^{6}$

\section{MEASUREMENTS AND RESULTS}

After implantation, the crystal lattice is heavily damaged, as expected, and most of the probe atoms are not located at substitutional sites. Thus, each probe atom is subjected to a different EFG. These EFGs strongly differ in magnitude and orientation in space, causing a quick breakdown of the anisotropy within the first $20 \mathrm{~ns}$ of the time window (Fig. 1, top row) independent of the $\mathrm{Si}$ concentration.

The recovery of the crystal lattice from the implantation-induced damage was monitored by taking PAC spectra at $293 \mathrm{~K}$ after annealing the samples for $120 \mathrm{~s}$ at several temperatures from $600 \mathrm{~K}$ up to $1400 \mathrm{~K}$. Distinct differences depending on the Si concentration could be observed. This can be seen most clearly in Fig. 2, where the spectra obtained from the undoped sample and the one with the highest $\mathrm{Si}$ concentration are compared. The unique $\mathrm{QI} v_{\mathrm{Q}} \approx 6 \mathrm{MHz}$ expected for In on a Ga site in $\mathrm{GaN}^{7}$ develops more clearly in the highly doped sample (annealing temperatures above $600 \mathrm{~K}$ in Fig. 2) and reaches its maximum after annealing at $1200 \mathrm{~K}$ (Fig. 2 top). Here, a least-squares fit to the data yields a fraction of $87(1) \%$ of the probe atoms on the regular lattice site as compared with $62(1) \%$ for the undoped sample. Even after the $1400 \mathrm{~K}$ step, the highly doped sample still shows 44(1)\% of In on this site, whereas in the undoped sample the QI has disappeared completely and instead a largely undisturbed spectrum is found. The undisturbed spectrum is a well-known phenomenon which occurs when nitrogen is completely lost from the top crystal layers and the In probe atoms are dissolved in the remaining liquid $\mathrm{Ga}$ drops. ${ }^{8}$ The different behavior of Si-doped and undoped sample was verified by taking pictures of the sample surface by optical microscopy, where larger and more numerous Ga droplets were found on the undoped sample. A possible explanation for this effect might be the 

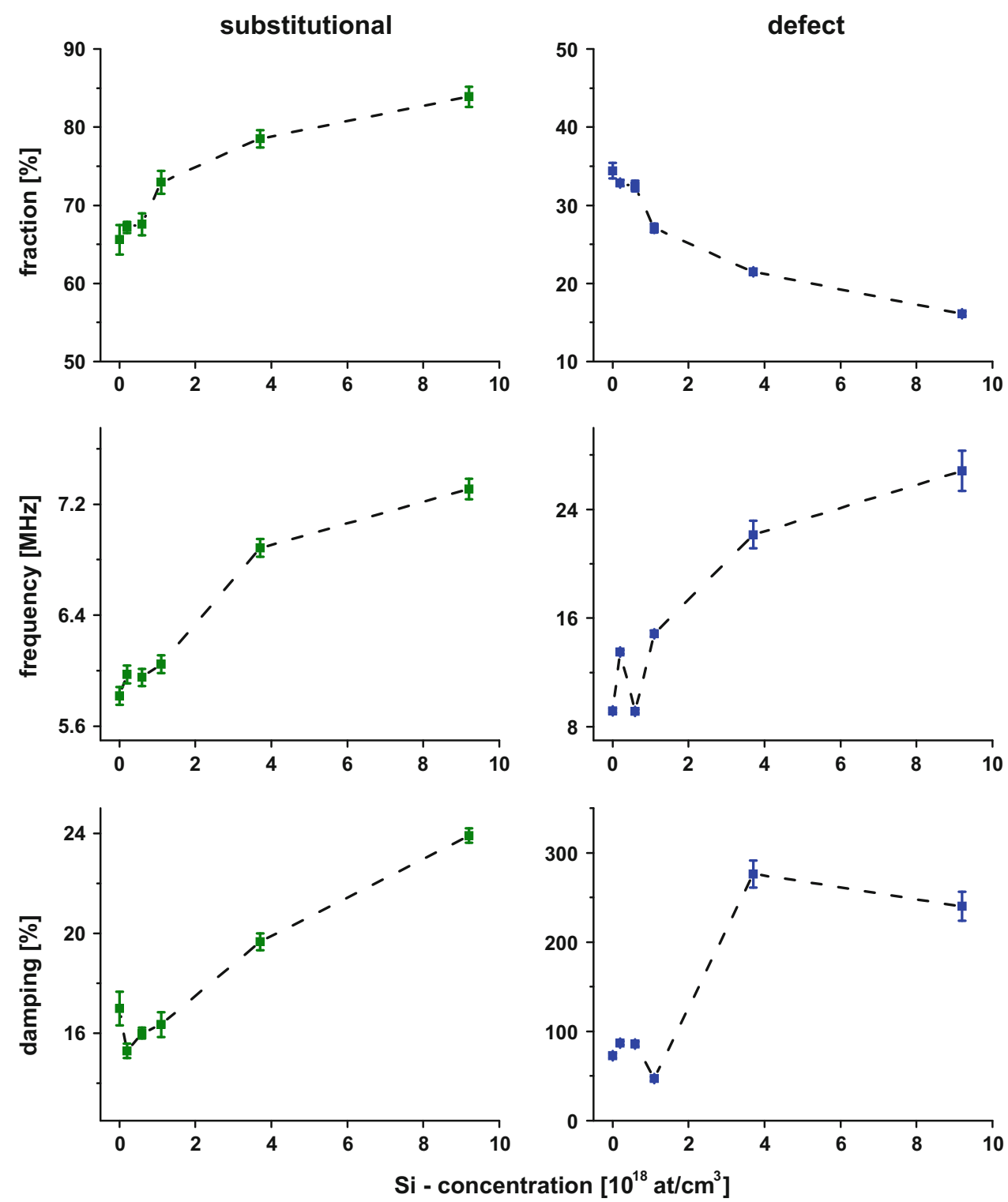

Fig. 3. Parameters derived from a least-squares fit to $A_{22}(t)$ spectra taken at elevated sample temperatures for the substitutional and defectassociated site. Lines are inserted to guide the eye.

formation of strong $\mathrm{Si}-\mathrm{N}$ bonds as in silicon nitride, which prevent nitrogen from diffusing out of the sample with high Si content.

Measurements with different sample orientations confirmed that in all samples the principal component of the EFG is axially symmetric $(\eta=0)$ and parallel to the $c$-axis of the crystal as observed in previous studies. ${ }^{7}$

At room temperature, after annealing at $1200 \mathrm{~K}$, the EFG increases with the silicon concentration (Fig. 3). This can be due to a change of the $u$ parameter, caused by the incorporation of silicon atoms, or an increase of the charge carrier density. ${ }^{9}$

Temperature-dependent measurements up to $900 \mathrm{~K}$ showed that the increase of the EFG with temperature is less pronounced when doping with concentrations larger than $3.7 \times 10^{18} \mathrm{Si} / \mathrm{cm}^{3}$.
Furthermore, compared with undoped GaN, lower damping of the QI frequency is observed up to a concentration of $1.1 \times 10^{18} \mathrm{Si} / \mathrm{cm}^{3}$ at room temperature. These results can be attributed to a reduced dislocation density, caused by the silicon doping. ${ }^{10}$ This reduction of the damping is enhanced at higher temperatures. At $900 \mathrm{~K}$, up to $3.7 \times 10^{18} \mathrm{Si} / \mathrm{cm}^{3}$, lower damping than for undoped $\mathrm{GaN}$ is measured.

In all samples, a minor fraction of probe atoms subjected to a much larger EFG is observed in the PAC spectra. This fraction is attributed to a nitrogen vacancy, trapped by the oversized In probe atoms on the nearest $\mathrm{N}$ site in $c$-direction. ${ }^{11,12} \mathrm{At}$ room temperature, this fraction decreases with the silicon concentration. Since in recent density functional calculations by Ganchenkova and Nieminen ${ }^{13}$ it was found that in $n$-type GaN negatively charged 
nitrogen vacancies are the dominating defect, it is most probable that ionized donors, in this case $\mathrm{Si}^{+}$, act as competing traps for $\mathrm{N}$ vacancies.

\section{CONCLUSIONS}

The annealing behavior of Si-doped GaN was studied after implantation of various doses of In. By means of the perturbed angular correlation technique applied to the nuclear probe ${ }^{111}$ In it could be shown that the optimum annealing condition was $1200 \mathrm{~K}$ for $120 \mathrm{~s}$ under nitrogen atmosphere. Large Si concentrations in the implanted GaN films were found to effectively stabilize the GaN surface at high annealing temperatures.

\section{REFERENCES}

1. H. Frauenfelder and R.M. Steffen, Alpha, Beta, and Gamma-Ray Spectroscopy, ed. K. Siegbahn (Amsterdam, The Netherlands: North-Holland, 1965).

2. H. Koch, Defekt-Fremdatom Wechselwirkung in den hexagonalen Metallen Rhenium und Lutetium $(\mathrm{PhD}$ thesis, Universität Bonn, 1992).
3. R. Valentini, Winkelkorrelationsuntersuchungen an Seltenen Erden in Halbleiter mit großer Bandlücke ( $\mathrm{PhD}$ thesis, Universität Bonn, 2011).

4. K. Köhler, J. Wiegert, H.P. Menner, M. Maier, and L. Kirste, J. Appl. Phys. 103, 023706 (2008).

5. J.F. Ziegler, J.P. Biersack, and U. Littmark, The Stopping and Range of Ions in Solids (New York: Pergamon, 1985).

6. M. Forker, W. Herz, U. Hütten, R. Müßeler, J. Schmidberger, D. Simon, A. Weingarten, and S.C. Bedi, Nuclear Instrum. Methods A327, 456 (1993).

7. K. Lorenz, F. Ruske, and R. Vianden, Appl. Phys. Lett. 80, 4531 (2002).

8. G. Marx, Akzeptor-Wasserstoff-Komplexe und spannungsinduzierte elektrische Feldgradienten in Silizium und Germanium (PhD thesis, Universität Bonn, 1995).

9. M. Corti, A. Gabetta, M. Fanciulli, A. Svane, and N.E. Christensen, Phys. Rev. B 67, 064416 (2003).

10. S. Ruvimov, Z. Liliental-Weber, T. Suski, J.W. Ager III, J. Washburn, J. Krueger, C. Kisielowski, E.R. Weber, H. Amano, and I. Akasaki, Appl. Phys. Lett. 69, 990 (1996).

11. P. Pyykkö, Phys. Rev. B 85, 24115 (2012).

12. P. Keßler, K. Lorenz, and R. Vianden, Defect Diffusion Forum 311, 167 (2011).

13. M.G. Ganchenkova and R.M. Nieminen, Phys. Rev. Lett. 96, 196402 (2006). 